УДК 519.85

MSC 47J20, 49J40, 65K15, 90C25

\title{
FINITE CONVERGENCE OF TWO-STAGE ALGORITHMS FOR SOLVING OF EQUILIBRIUM PROBLEMS
}

\author{
Ya. I. Vedel, E. N. Golubeva, V. V. Semenov
}

Faculty of Computer Science and Cybernetics, Taras Shevchenko Kiev National University, Kiev, Ukraine, E-mail: yana.vedel@gmail.com, Katrin_G@bigmir.net, volodya.semenov@gmail.com

\section{КОНЕЧНОЕ ЧИСЛО ИТЕРАЦИЙ В ДВУХЭТАПНЫХ АЛГОРИТМАХ ДЛЯ РЕШЕНИЯ ЗАДАЧ О РАВНОВЕСИИ}

\author{
Я. И. ВеДЕЛЬ, Е. Н. ГоЛУБевА, В. В. СЕмёнов
}

Факультет компьютерных наук и кибернетики, Киевский национальный университет имени Тараса Шевченко, Киев, Украина, E-mail: yana.vedel@gmail.com,

Katrin_G@bigmir.net, volodya.semenov@gmail.com

\begin{abstract}
A two iterative two-stage proximal algorithms for the approximate solution of the equilibrium problem in a Hilbert space is considered. In this article we proved the convergence of algorithms in a finite number of iterations when the condition of sharpness is fulfilled.

KEYWORDS: equilibrium problem, bifunction, pseudo-monotonicity, sharpness, two-stage proximal algorithm, Hilbert space, finite convergence.

АннотАция. Рассмотрены два двухэтапных проксимальных алгоритма приближенного решения задачи о равновесии в гильбертовом пространстве. В работе доказана сходимость алгоритмов к решению за конечное число итераций при выполнении условия остроты.

КЛЮЧЕВЫЕ СЛОВА: Задача о равновесии, бифункция, псевдомонотонность, условие остроты, двухэтапный проксимальный алгоритм, гильбертово пространство, конечная сходимость.
\end{abstract}

\section{ВВЕДЕНИЕ}

Популярным направлением современного прикладного нелинейного анализа является исследование задач о равновесии (неравенств Ки Фаня, задач равновесного программирования) вида [1-3]

$$
\text { найти } x \in C: F(x, y) \geq 0 \quad \forall y \in C \text {, }
$$


где $C$ - непустое подмножество гильбертова пространства $H, F: C \times C \rightarrow$ $\mathbb{R}$ - бифункция. В виде (1) можно сформулировать задачи математического программирования, вариационные неравенства и многие игровые задачи. Приведем три типичные формулировки.

(1) Если $F(x, y)=\varphi(y)-\varphi(x)$, где $\varphi: C \rightarrow \mathbb{R}$, то задача (1) является задачей условной минимизации

$$
\varphi \rightarrow \min _{C}
$$

(2) Если $F(x, y)=(A x, y-x)$, где $A: C \rightarrow H$, то задача (1) сводится к классическому вариационному неравенству

$$
\text { найти } x \in C:(A x, y-x) \geq 0 \forall y \in C \text {. }
$$

(3) Пусть $I-$ конечное множество индексов. Для каждого $i \in I$ заданы множество $C_{i}$ и функция $\varphi_{i}: C \rightarrow \mathbb{R}$, где $C=\prod_{i \in I} C_{i}$. Для $x=$ $\left(x_{i}\right)_{i \in I} \in C$ обозначим $x^{i}=\left(x_{j}\right)_{j \in I, j \neq i}$. Точку $\bar{x}=\left(\bar{x}_{i}\right)_{i \in I}$ называют равновесием Нэша, если для всех $i \in I$ выполняются неравенства

$$
\varphi_{i}(\bar{x}) \leq \varphi_{i}\left(\bar{x}^{i}, y_{i}\right) \quad \forall y_{i} \in C_{i} .
$$

Определим функцию $F: C \times C \rightarrow \mathbb{R}$ следующим образом

$$
F(x, y)=\sum_{i \in I}\left(\varphi_{i}\left(x^{i}, y_{i}\right)-\varphi_{i}(x)\right) .
$$

Точка $\bar{x} \in C$ является равновесием Нэша тогда и только тогда, когда она является решением задачи (1).

Алгоритмам решения равновесных и близких задач посвящено большое количество работ. Частным случаем задач о равновесии являются вариационные неравенства. Для их решения Г. М. Корпелевич предложила экстраградиентный метод [4]. Аналогам экстраградиентного метода для задач о равновесии посвящены работы $[1,5,6]$. В 1980 Л. Д. Попов [7] предложил для поиска седловых точек выпукло-вогнутых функций, определенных в конечномерном евклидовом пространстве, интересную модификацию метода Эрроу-Гурвица. В статье [8] был предложен двухэтапный проксимальный алгоритм для решения задач о равновесии в гильбертовом пространстве, являющийся адаптацией метода Л. Д. Попова к общим задачам равновесного программирования (см. также [9-11]).

В большинстве работ доказывается слабая или сильная сходимость к решению, но при дополнительных условиях остроты (sharpness condition) для некоторых алгоритмов решения вариационных неравенств удается показать сходимость к решению за конечное число итераций [12-23].

В данной статье, продолжающей работы $[11,23]$, исследуется сходимость к решению за конечное число итераций двухэтапных алгоритмов приближенного решения задачи о равновесии в гильбертовом пространстве.

\section{1. ЗАДАЧА О РАВНОВЕСИИ И УСЛОВИЕ ОСТРОТЫ}

Всюду далее $H$ - действительное гильбертово пространство со скалярным произведением $(\cdot, \cdot)$ и порожденной нормой $\|\cdot\|$. 
Для непустого выпуклого замкнутого множества $C \subseteq H$ и бифункции $F: C \times C \rightarrow \mathbb{R}$ рассмотрим задачу о равновесии:

$$
\text { найти } x \in C: F(x, y) \geq 0 \quad \forall y \in C \text {. }
$$

Будем предполагать выполненными следующие условия:

(A1) $F(x, x)=0$ для всех $x \in C$;

(A2) для всех $x, y \in C$ из $F(x, y) \geq 0$ следует $F(y, x) \leq 0$ (псевдомонотонность);

(A3) для всех $x \in C$ функция $F(x, \cdot)$ полунепрерывна снизу и выпукла на $C$;

(A4) для всех $y \in C$ функция $F(\cdot, y)$ полунепрерывна сверху на $C$;

(A5) для всех $x, y, z \in C$ имеет место

$$
F(x, y) \leq F(x, z)+F(z, y)+d\|x-z\|\|z-y\|,
$$

где $d-$ положительная константа (липшицевость).

Замечание 1. Условие (А5) типа липшицевости сильнее известного условия G. Mastroeni [2].

Рассмотрим так называемую дуальную [24] (для задачи (3)) задачу о равновесии:

$$
\text { найти } x \in C: F(y, x) \leq 0 \quad \forall y \in C \text {. }
$$

Множества решений задач (3) и (4) обозначим $S$ и $S^{*}$. При выполнении условий (A1)-(А4) имеем $S=S^{*}[24]$. В частности, множество $S-$ выпуклое и замкнутое, поскольку

$$
S=S^{*}=\bigcap_{y \in C}\{x \in C: F(y, x) \leq 0\} .
$$

Далее будем предполагать, что $S \neq \emptyset$.

Будем рассматривать задачу (3), удовлетворяющую следующему условию остроты [23]

$$
\exists \alpha>0: F\left(x, P_{S} x\right) \leq-\alpha\left\|x-P_{S} x\right\| \quad \forall x \in C .
$$

где $P_{S}$ - оператор метрического проектирования на $S$.

Условие (5) для вариационного неравенства (2) принимает вид [12]

$$
\exists \alpha>0:\left(A x, x-P_{S} x\right) \geq \alpha\left\|x-P_{S} x\right\| \quad \forall x \in C,
$$

где $P_{S}$ - оператор метрического проектирования на $S$.

Для задач выпуклого программирования

$$
f \rightarrow \min _{C}
$$

в работах $[14-16,25]$ рассматривалось следующее понятие остроты минимума. Множество решений задачи минимизации является множеством острых минимумов, если выполняется неравенство

$$
\exists \alpha>0: f(x)-f\left(P_{S} x\right) \geq \alpha\left\|x-P_{S} x\right\| \quad \forall x \in C,
$$

где $S$ - множество решений исходной задачи (7). В [16] доказана сходимость к решению (7) за конечное число итераций проксимального метода 
и метода проекции градиента. В гладком случае из (8) следует (6) для равносильного (7) вариационного неравенства

$$
\text { найти } x \in C:(\nabla f(x), y-x) \geq 0 \quad \forall y \in C \text {. }
$$

Действительно, имеет место неравенство

$$
f(x)-f\left(P_{S} x\right) \leq\left(\nabla f(x), x-P_{S} x\right) \quad \forall x \in C .
$$

Откуда непосредственно следует желаемая импликация.

Конечная сходимость проксимального алгоритма для вариационных неравенств при выполнении условия остроты доказана в [20,21]. Аналогичные результаты получены для экстраградиентного метода [18] и двухшагового экстраградиентного метода [13]. В [23] конечная сходимость при выполнении условия остроты установлена для двухэтапного алгоритма Л. Д. Попова.

Пусть $g: H \rightarrow \mathbb{R} \cup\{+\infty\}$ - собственная выпуклая полунепрерывная снизу функция. Напомним, что проксимальным оператором [26], ассоциированным с функцией $g$, называют оператор

$$
H \ni x \mapsto \operatorname{prox}_{g} x=\operatorname{argmin}_{y \in \operatorname{dom} g}\left(g(y)+\frac{1}{2}\|y-x\|^{2}\right) \in \operatorname{dom} g .
$$

Оператор $\operatorname{prox}_{g}$ - твердо нерастягивающий (firmly nonexpansive) и

$$
g(y)-g(z)+(z-x, y-z) \geq 0 \forall y \in \operatorname{dom} g \Leftrightarrow z=\operatorname{prox}_{g} x .
$$

\section{2. ДВУХЭТАПНЫЕ АЛГОРИТМЫ И ВСПОМОГАТЕЛЬНЫЕ ФАКТЫ}

Рассмотрим следующие хорошо известные алгоритмы решения задачи о равновесии (3).

Алгоритм 1 (T. D. Quoc, L. D. Muu, N. V. Hien, [6]). Для $x_{1} \in C$ генерируем последовательность элементов $x_{n}, y_{n} \in C$ при помощи итерационной схемы

где $\lambda_{n}>0$.

$$
\left\{\begin{array}{l}
y_{n}=\operatorname{prox}_{\lambda_{n} F\left(x_{n}, \cdot\right)} x_{n} \\
x_{n+1}=\operatorname{prox}_{\lambda_{n} F\left(y_{n}, \cdot\right)} x_{n}
\end{array}\right.
$$

Алгоритм 2 (Я. И. Ведель, В. В. Семёнов, [8]). Для $x_{1}, y_{0} \in C$ генерируем последовательность элементов $x_{n}, y_{n} \in C$ при помощи итерационной схемы

$$
\left\{\begin{array}{l}
y_{n}=\operatorname{prox}_{\lambda_{n} F\left(y_{n-1}, \cdot\right)} x_{n}, \\
x_{n+1}=\operatorname{prox}_{\lambda_{n} F\left(y_{n}, \cdot\right)} x_{n}
\end{array}\right.
$$

где $\lambda_{n}>0$.

На каждом шаге алгоритмов следует решить две выпуклые задачи с сильно выпуклыми функциями. Предположим возможность их эффективного решения. 
Замечание 2. Если $F(x, y)=(A x, y-x)$, то алгориты принимают вид:

$$
\begin{aligned}
& \left\{\begin{array}{l}
x_{1} \in C, \\
y_{n}=P_{C}\left(x_{n}-\lambda_{n} A x_{n}\right), \\
x_{n+1}=P_{C}\left(x_{n}-\lambda_{n} A y_{n}\right),
\end{array}\right. \\
& \left\{\begin{array}{l}
x_{1} \in C, y_{0} \in C, \\
y_{n}=P_{C}\left(x_{n}-\lambda_{n} A y_{n-1}\right), \\
x_{n+1}=P_{C}\left(x_{n}-\lambda_{n} A y_{n}\right),
\end{array}\right.
\end{aligned}
$$

где $P_{C}$ - оператор метрического проектирования на множество $C$.

Первый алгоритм замечания 2 - экстраградиентный метод Г. М. Корпелевич. Частный случай второй схемы замечания 2 предложен российским математиком Л. Д. Поповым [7] для поиска седловых точек выпукло-вогнутых функций, определенных в конечномерном евклидовом пространстве. В работе [27] Ю. В. Малицкий и В. В. Семёнов доказали сходимость этого алгоритма для неравенств с монотонными и липшицевыми операторами, действующими в бесконечномерном гильбертовом пространстве, а также предложили его модификацию. А в работах $[28,29]$ предложены варианты метода с использованием брэгмановского расстояния вместо евклидового. Заметим, что в последнее время данный метод стал известен в среде специалистов по машинному обучению под названием «Extrapolation from the Past» [30].

Для последовательностей, порожденных алгоритмами 1 и 2 , имеют место следующие неравенства.

Лемма 1. Для порожденных алгоритмом 1 последовательностей $\left(x_{n}\right)$, $\left(y_{n}\right)$ и элемента $z \in S$ выполняется неравенство

$$
\begin{aligned}
\left\|x_{n+1}-z\right\|^{2} \leq\left\|x_{n}-z\right\|^{2}-\left(1-\lambda_{n} d\right) \| x_{n+1}- & y_{n} \|^{2}- \\
& -\left(1-\lambda_{n} d\right)\left\|y_{n}-x_{n}\right\|^{2} .
\end{aligned}
$$

Доказательство. Поскольку $x_{n+1}=\operatorname{prox}_{\lambda_{n} F\left(y_{n}, \cdot\right)} x_{n}$, то имеем

$$
\lambda_{n} F\left(y_{n}, y\right)-\lambda_{n} F\left(y_{n}, x_{n+1}\right)+\left(x_{n+1}-x_{n}, y-x_{n+1}\right) \geq 0 \quad y \in C .
$$

Положив в (10) $y=z \in S$ и учтя неравенство $F\left(y_{n}, z\right) \leq 0$ (следует из псевдомонотонности $F$ ), получим

$$
\left(x_{n+1}-x_{n}, z-x_{n+1}\right) \geq \lambda_{n} F\left(y_{n}, x_{n+1}\right) .
$$

Поскольку

$$
F\left(y_{n}, x_{n+1}\right) \geq F\left(x_{n}, x_{n+1}\right)-F\left(x_{n}, y_{n}\right)-d\left\|x_{n}-y_{n}\right\|\left\|y_{n}-x_{n+1}\right\|,
$$

то из (11) следует неравенство

$$
\begin{aligned}
\left(x_{n+1}-x_{n}, z-x_{n+1}\right) \geq \lambda_{n} F\left(x_{n}, x_{n+1}\right) & -\lambda_{n} F\left(x_{n}, y_{n}\right)- \\
& -\lambda_{n} d\left\|x_{n}-y_{n}\right\|\left\|y_{n}-x_{n+1}\right\| .
\end{aligned}
$$

Поскольку $y_{n}=\operatorname{prox}_{\lambda_{n} F\left(x_{n}, \cdot\right)} x_{n}$ и $x_{n+1} \in C$, то имеем

$$
\lambda_{n} F\left(x_{n}, x_{n+1}\right)-\lambda_{n} F\left(x_{n}, y_{n}\right)+\left(y_{n}-x_{n}, x_{n+1}-y_{n}\right) \geq 0 .
$$


Учитывая (13) в (12), приходим к неравенству

$$
\begin{aligned}
\left(x_{n+1}-x_{n}, z-x_{n+1}\right) \geq\left(y_{n}-x_{n}, y_{n}-\right. & \left.x_{n+1}\right)- \\
& -\lambda_{n} d\left\|x_{n}-y_{n}\right\|\left\|y_{n}-x_{n+1}\right\| .
\end{aligned}
$$

Имеет место равенство

$$
2\left(x_{n+1}-x_{n}, z-x_{n+1}\right)=\left\|x_{n}-z\right\|^{2}-\left\|x_{n+1}-x_{n}\right\|^{2}-\left\|x_{n+1}-z\right\|^{2} .
$$

Поэтому (14) можно переписать в виде

$$
\begin{aligned}
& \left\|x_{n}-z\right\|^{2}-\left\|x_{n+1}-x_{n}\right\|^{2}-\left\|x_{n+1}-z\right\|^{2} \geq \\
& \quad \geq 2\left(y_{n}-x_{n}, y_{n}-x_{n+1}\right)-2 \lambda_{n} d\left\|x_{n}-y_{n}\right\|\left\|y_{n}-x_{n+1}\right\| .
\end{aligned}
$$

Откуда

$$
\begin{aligned}
\left\|x_{n+1}-z\right\|^{2} \leq\left\|x_{n}-z\right\|^{2}-\| x_{n+1}- & x_{n} \|^{2}-2\left(y_{n}-x_{n}, y_{n}-x_{n+1}\right)+ \\
& +\lambda_{n} d\left\|x_{n}-y_{n}\right\|^{2}+\lambda_{n} d\left\|y_{n}-x_{n+1}\right\|^{2} .
\end{aligned}
$$

Поскольку

$$
\left\|x_{n+1}-x_{n}\right\|^{2}=\left\|x_{n+1}-y_{n}\right\|^{2}+\left\|y_{n}-x_{n}\right\|^{2}+2\left(y_{n}-x_{n}, x_{n+1}-y_{n}\right),
$$

то

$$
\begin{aligned}
& \left\|x_{n+1}-z\right\|^{2} \leq\left\|x_{n}-z\right\|^{2}-\left\|x_{n+1}-y_{n}\right\|^{2}-\left\|y_{n}-x_{n}\right\|^{2}+ \\
& +\lambda_{n} d\left\|x_{n}-y_{n}\right\|^{2}+\lambda_{n} d\left\|y_{n}-x_{n+1}\right\|^{2}=\left\|x_{n}-z\right\|^{2}- \\
& \quad-\left(1-\lambda_{n} d\right)\left\|x_{n}-y_{n}\right\|^{2}-\left(1-\lambda_{n} d\right)\left\|y_{n}-x_{n+1}\right\|^{2},
\end{aligned}
$$

что и требовалось доказать.

Лемма 2. Для порожденных алгоритмом 2 последовательностей $\left(x_{n}\right)$, $\left(y_{n}\right)$ и элемента $z \in S$ выполняется неравенство

$$
\begin{aligned}
\left\|x_{n+1}-z\right\|^{2} \leq\left\|x_{n}-z\right\|^{2}-\left(1-\lambda_{n} d\right)\left\|x_{n+1}-y_{n}\right\|^{2}- \\
-\left(1-2 \lambda_{n} d\right)\left\|y_{n}-x_{n}\right\|^{2}+2 \lambda_{n} d\left\|x_{n}-y_{n-1}\right\|^{2} .
\end{aligned}
$$

Доказательство. Имеем

$$
\begin{aligned}
\left\|x_{n+1}-z\right\|^{2}= & \left\|x_{n}-z\right\|^{2}-\left\|x_{n}-x_{n+1}\right\|^{2}+2\left(x_{n+1}-x_{n}, x_{n+1}-z\right)= \\
= & \left\|x_{n}-z\right\|^{2}-\left\|x_{n}-y_{n}\right\|^{2}-\left\|y_{n}-x_{n+1}\right\|^{2}- \\
& -2\left(x_{n}-y_{n}, y_{n}-x_{n+1}\right)+2\left(x_{n+1}-x_{n}, x_{n+1}-z\right) .
\end{aligned}
$$

Из определения точек $x_{n+1}$ и $y_{n}$ следует

$$
\begin{gathered}
\lambda_{n} F\left(y_{n}, z\right)-\lambda_{n} F\left(y_{n}, x_{n+1}\right) \geq\left(x_{n+1}-x_{n}, x_{n+1}-z\right), \\
\lambda_{n} F\left(y_{n-1}, x_{n+1}\right)-\lambda_{n} F\left(y_{n-1}, y_{n}\right) \geq-\left(x_{n}-y_{n}, y_{n}-x_{n+1}\right) .
\end{gathered}
$$

Использовав неравенства (17), (18) для оценки скалярных произведений в (16), получаем

$$
\begin{aligned}
\left\|x_{n+1}-z\right\|^{2} \leq\left\|x_{n}-z\right\|^{2}-\left\|x_{n}-y_{n}\right\|^{2}-\left\|y_{n}-x_{n+1}\right\|^{2}+ \\
+2 \lambda_{n}\left\{F\left(y_{n}, z\right)-F\left(y_{n}, x_{n+1}\right)+F\left(y_{n-1}, x_{n+1}\right)-F\left(y_{n-1}, y_{n}\right)\right\} .
\end{aligned}
$$


Из псевдомонотонности бифункции $F$ и включения $z \in S$ следует

$$
F\left(y_{n}, z\right) \leq 0,
$$

а липшицевость $F$ гарантирует выполнение неравенства

$$
\begin{aligned}
& -F\left(y_{n}, x_{n+1}\right)+F\left(y_{n-1}, x_{n+1}\right)-F\left(y_{n-1}, y_{n}\right) \leq \\
& \quad \leq d\left\|y_{n-1}-y_{n}\right\|\left\|y_{n}-x_{n+1}\right\| \leq \frac{d}{2}\left\|y_{n-1}-y_{n}\right\|^{2}+\frac{d}{2}\left\|y_{n}-x_{n+1}\right\|^{2} .
\end{aligned}
$$

Использовав вышеприведенные оценки в (19), получаем

$$
\begin{aligned}
\left\|x_{n+1}-z\right\|^{2} \leq\left\|x_{n}-z\right\|^{2}- & \left\|x_{n}-y_{n}\right\|^{2}-\left\|y_{n}-x_{n+1}\right\|^{2}+ \\
& +\lambda_{n} d\left\|y_{n-1}-y_{n}\right\|^{2}+\lambda_{n} d\left\|y_{n}-x_{n+1}\right\|^{2} .
\end{aligned}
$$

Член $\left\|y_{n-1}-y_{n}\right\|^{2}$ оценим следующим образом

$$
\left\|y_{n-1}-y_{n}\right\|^{2} \leq 2\left\|y_{n-1}-x_{n}\right\|^{2}+2\left\|y_{n}-x_{n}\right\|^{2} .
$$

Учтя эту оценку в (20), приходим к неравенству

$$
\begin{aligned}
\left\|x_{n+1}-z\right\|^{2} & \leq\left\|x_{n}-z\right\|^{2}-\left\|x_{n}-y_{n}\right\|^{2}-\left\|y_{n}-x_{n+1}\right\|^{2}+ \\
& +2 \lambda_{n} d\left\|y_{n-1}-x_{n}\right\|^{2}+2 \lambda_{n} d\left\|y_{n}-x_{n}\right\|^{2}+\lambda_{n} d\left\|y_{n}-x_{n+1}\right\|^{2},
\end{aligned}
$$

то есть к неравенству (15).

Из лемм 1 и 2 можно извлечь следующие факты об асимптотическом поведении порожденных алгоритмами последовательностей.

Лемма 3. Пусть $\lambda_{n} \in[\underline{\lambda}, \bar{\lambda}] \subseteq\left(0, \frac{1}{d}\right)$. Тогда для порожденных алгоритмом 1 последовательностей $\left(x_{n}\right),\left(y_{n}\right)$ имеет место

$$
\left\|x_{n+1}-x_{n}\right\| \rightarrow 0,\left\|x_{n+1}-y_{n}\right\| \rightarrow 0 .
$$

Доказательство. Из неравенства (9) получаем

$$
\sum_{n}\left\|y_{n}-x_{n}\right\|^{2}<+\infty, \quad \sum_{n}\left\|x_{n+1}-y_{n}\right\|^{2}<+\infty .
$$

Следовательно,

$$
\lim _{n \rightarrow \infty}\left\|x_{n+1}-y_{n}\right\|=\lim _{n \rightarrow \infty}\left\|x_{n}-y_{n}\right\|=0 .
$$

Из неравенства

$$
\left\|x_{n+1}-x_{n}\right\| \leq\left\|x_{n+1}-y_{n}\right\|+\left\|y_{n}-x_{n}\right\|
$$

получаем

$$
\lim _{n \rightarrow \infty}\left\|x_{n+1}-x_{n}\right\|=0
$$

чем и завершаем доказательство.

Лемма 4. Пусть $\lambda_{n} \in[\underline{\lambda}, \bar{\lambda}] \subseteq\left(0, \frac{1}{3 d}\right)$. Тогда для порожденных алгоритмом 2 последовательностей $\left(x_{n}\right),\left(y_{n}\right)$ имеет место

$$
\left\|x_{n}-y_{n}\right\| \rightarrow 0,\left\|y_{n}-y_{n-1}\right\| \rightarrow 0,\left\|x_{n+1}-x_{n}\right\| \rightarrow 0,\left\|x_{n+1}-y_{n}\right\| \rightarrow 0 .
$$


Доказательство. Пусть $z \in S$. Положим

$$
\begin{aligned}
& a_{n}=\left\|x_{n}-z\right\|^{2}+2 \lambda_{n} d\left\|y_{n-1}-x_{n}\right\|^{2}, \\
& b_{n}=\left(1-2 \lambda_{n} d\right)\left\|y_{n}-x_{n}\right\|^{2}+\left(1-3 \lambda_{n} d\right)\left\|y_{n}-x_{n+1}\right\|^{2} .
\end{aligned}
$$

Тогда (15) принимает вид

$$
a_{n+1} \leq a_{n}-b_{n}
$$

Существует предел

$$
\lim _{n \rightarrow \infty}\left(\left\|x_{n}-z\right\|^{2}+2 \lambda_{n} d\left\|y_{n-1}-x_{n}\right\|^{2}\right)
$$

и

$$
\lim _{n \rightarrow \infty}\left(\left(1-2 \lambda_{n} d\right)\left\|y_{n}-x_{n}\right\|^{2}+\left(1-3 \lambda_{n} d\right)\left\|y_{n}-x_{n+1}\right\|^{2}\right)=0 .
$$

Откуда получаем

$$
\lim _{n \rightarrow \infty}\left\|y_{n}-x_{n}\right\|=\lim _{n \rightarrow \infty}\left\|y_{n}-x_{n+1}\right\|=0 .
$$

Из неравенств

$$
\begin{aligned}
\left\|x_{n+1}-x_{n}\right\| & \leq\left\|x_{n+1}-y_{n}\right\|+\left\|y_{n}-x_{n}\right\|, \\
\left\|y_{n}-y_{n-1}\right\| & \leq\left\|y_{n}-x_{n}\right\|+\left\|x_{n}-y_{n-1}\right\|
\end{aligned}
$$

получаем

$$
\lim _{n \rightarrow \infty}\left\|x_{n+1}-x_{n}\right\|=\lim _{n \rightarrow \infty}\left\|y_{n}-y_{n-1}\right\|=0,
$$

чем и завершаем доказательство.

Из лемм 3 и 4 следует слабая сходимость алгоритмов 1 и 2 [11]. Далее перейдем основному результату работы - теоремам о поведении алгоритмов 1 и 2 при выполнении условия остроты (5).

\section{3. ТЕОРЕМЫ О КОНЕЧНОЙ СХОДИМОСТИ}

Рассмотрим пару последовательностей $\left(x_{n}\right),\left(y_{n}\right)$, таких, что

$$
x_{n+1}=\operatorname{prox}_{\lambda_{n} F\left(y_{n}, \cdot\right)} x_{n}, y_{n} \in C,
$$

причем $\lambda_{n} \geq \underline{\lambda}>0 \forall n \in \mathbb{N}$ и

$$
\lim _{n \rightarrow \infty}\left\|x_{n+1}-x_{n}\right\|=\lim _{n \rightarrow \infty}\left\|x_{n+1}-y_{n}\right\|=0 .
$$

Покажем, что при выполнении условия остроты (5) для задачи о равновесии (3) последовательность $\left(x_{n}\right)$, сходится к некоторому решению (3) за конечное число итераций, т.е. существует номер $n \in \mathbb{N}$ такой, что $x_{i} \in S$ для всех $i \geq n$.

Равенство

$$
x_{n+1}=\operatorname{prox}_{\lambda_{n} F\left(y_{n}, \cdot\right)} x_{n}
$$

равносильно неравенству

$$
\frac{\left(x_{n+1}-x_{n}, y-x_{n+1}\right)}{\lambda_{n}}+F\left(y_{n}, y\right)-F\left(y_{n}, x_{n+1}\right) \geq 0 \quad \forall y \in C .
$$


Имеем

$$
\begin{aligned}
\frac{\left(x_{n+1}-x_{n}, y-x_{n+1}\right)}{\lambda_{n}} & +F\left(x_{n+1}, y\right)- \\
& -F\left(x_{n+1}, y\right)+F\left(y_{n}, y\right)-F\left(y_{n}, x_{n+1}\right) \geq 0 \quad \forall y \in C .
\end{aligned}
$$

Откуда получаем

$$
\begin{aligned}
-F\left(x_{n+1}, y\right) \leq & \frac{\left(x_{n+1}-x_{n}, y-x_{n+1}\right)}{\lambda_{n}}- \\
& -F\left(x_{n+1}, y\right)+F\left(y_{n}, y\right)-F\left(y_{n}, x_{n+1}\right) \leq \\
& \leq \frac{\left\|x_{n+1}-x_{n}\right\|\left\|y-x_{n+1}\right\|}{\lambda_{n}}+d\left\|y_{n}-x_{n+1}\right\|\left\|y-x_{n+1}\right\| .
\end{aligned}
$$

Предположим, что существует подпоследовательность $\left(x_{n_{k}}\right)$ такая, что

$$
x_{n_{k}} \notin S \quad \forall k \in \mathbb{N} .
$$

Пусть $p_{n_{k}}=P_{S} x_{n_{k}}$. Имеем

$$
F\left(x_{n_{k}}, p_{n_{k}}\right) \leq-\alpha\left\|x_{n_{k}}-p_{n_{k}}\right\| .
$$

Используя $(22)$ в $(21)$, получаем

$$
\alpha\left\|x_{n_{k}}-p_{n_{k}}\right\| \leq \frac{\left\|x_{n_{k}}-x_{n_{k}-1}\right\|\left\|p_{n_{k}}-x_{n_{k}}\right\|}{\lambda_{n_{k}-1}}+d\left\|y_{n_{k}-1}-x_{n_{k}}\right\|\left\|p_{n_{k}}-x_{n_{k}}\right\| .
$$

Откуда

$$
\alpha \leq \frac{\left\|x_{n_{k}}-x_{n_{k}-1}\right\|}{\lambda_{n_{k}-1}}+d\left\|y_{n_{k}-1}-x_{n_{k}}\right\|=o(1)
$$

что противоречит условию $\alpha>0$. Таким образом, $x_{n} \in S$ для всех достаточно больших номеров $n$.

Из приведенного рассуждения и леммы 3 вытекает следующая теорема.

Теорема 1. Пусть $H-$ гильбертово пространство, $C \subseteq H-$ непустое выпуклое замкнутое множество, для бифункиии $F: C \times C \rightarrow \mathbb{R}$ выполнены условия (A1)-(A5) и $S \neq \emptyset$. Предположим, что выполнено условие остроты (5) и $\lambda_{n} \in[\underline{\lambda}, \bar{\lambda}] \subseteq\left(0, \frac{1}{d}\right)$. Тогда последовательности $\left(x_{n}\right),\left(y_{n}\right)$, генерируемые алгоритмом 1 , сходятся $к$ некоторому решению задачи о равновесии (3) за конечное число итераций, т.е. существует номер $n \in \mathbb{N}$ mакой, что $x_{n}=y_{n} \in S$.

Доказательство. Имеем $x_{n} \in S$ для всех достаточно больших номеров $n$. А из $y_{n}=\operatorname{prox}_{\lambda_{n} F\left(x_{n}, \cdot\right)} x_{n}$ и $x_{n} \in S$ следует $x_{n}=y_{n}$.

Аналогично доказывается наш второй основной результат.

Теорема 2. Пусть $H-$ гильбертово пространство, $C \subseteq H-$ непустое выпуклое замкнутое множество, для бифункиии $F: C \times C \rightarrow \mathbb{R}$ выполнены условия (A1)-(A5) и $S \neq \emptyset$. Предположсим, что выполнено условие остроты (5) и $\lambda_{n} \in[\underline{\lambda}, \bar{\lambda}] \subseteq\left(0, \frac{1}{3 d}\right)$. Тогда последовательности $\left(x_{n}\right),\left(y_{n}\right)$, генерируемые алгоритмом 2, сходятся к решениям задачи о равновесии (3) за конечное число итераций, т.е. существует номер $N \in \mathbb{N}$ такой, что $x_{n}, y_{n} \in S$ для всех $n \geq N$. 
Доказательство. Рассмотрим равенство

$$
y_{n}=\operatorname{prox}_{\lambda_{n} F\left(y_{n-1}, \cdot\right)} x_{n} .
$$

Оно равносильно неравенству

$$
\frac{\left(y_{n}-x_{n}, y-y_{n}\right)}{\lambda_{n}}+F\left(y_{n-1}, y\right)-F\left(y_{n-1}, y_{n}\right) \geq 0 \quad \forall y \in C .
$$

Откуда получаем

$$
\begin{aligned}
& -F\left(y_{n}, y\right) \leq \frac{\left(y_{n}-x_{n}, y-y_{n}\right)}{\lambda_{n}}- \\
& -F\left(y_{n}, y\right)+F\left(y_{n-1}, y\right)-F\left(y_{n-1}, y_{n}\right) \leq \\
& \quad \leq \frac{\left\|y_{n}-x_{n}\right\|\left\|y-y_{n}\right\|}{\lambda_{n}}+d\left\|y_{n}-y\right\|\left\|y_{n-1}-y_{n}\right\| .
\end{aligned}
$$

Предположим, что существует подпоследовательность $\left(y_{n_{k}}\right)$ такая, что

$$
y_{n_{k}} \notin S \quad \forall k \in \mathbb{N} .
$$

Пусть $m_{n_{k}}=P_{S} y_{n_{k}}$. Имеем

$$
F\left(y_{n_{k}}, m_{n_{k}}\right) \leq-\alpha\left\|y_{n_{k}}-m_{n_{k}}\right\| .
$$

Используя (24) в (23), получаем

$$
\alpha\left\|y_{n_{k}}-m_{n_{k}}\right\| \leq \frac{\left\|y_{n_{k}}-x_{n_{k}}\right\|\left\|m_{n_{k}}-y_{n_{k}}\right\|}{\lambda_{n_{k}}}+d\left\|y_{n_{k}-1}-y_{n_{k}}\right\|\left\|m_{n_{k}}-y_{n_{k}}\right\| .
$$

Откуда (учитывая лемму 4) получаем

$$
\alpha \leq \frac{\left\|y_{n_{k}}-x_{n_{k}}\right\|}{\lambda_{n_{k}}}+d\left\|y_{n_{k}-1}-y_{n_{k}}\right\|=o(1)
$$

что противоречит условию $\alpha>0$. Таким образом, $y_{n} \in S$ для всех достаточно больших номеров $n$.

Объединив с ранее полученным приходим к утверждению теоремы.

\section{ЗАКЛЮЧЕНИЕ}

В работе рассмотрены два двухэтапных проксимальных алгоритма приближенного решения задачи о равновесии в гильбертовом пространстве. Первый алгоритм - предложенное в [6], обобщение экстраградиентного метода Г. М. Корпелевич. А второй алгоритм был предложен в работе [8] и является развитием модификации Л. Д. Попова [7] схемы Эрроу-Гурвица поиска седловых точек выпукло-вогнутых функций. В работе доказана сходимость алгоритмов к решению за конечное число итераций при выполнении условия остроты.

Работа выполнена при поддержке Министерства образования и науки Украины (проект «Математичне моделювання та оптимізація динамічних систем для оборони, медицини та екології, 0219U008403) и Национальной академии наук Украины (проект «Нові методи дослідження коректності та розв'язання задач дискретної оптимізації, варіаційних нерівностей та їх застосування», 0119U101608). 


\section{ЛитеРАТУРА}

1. Antipin A. S. Equilibrium programming: Proximal methods. Comput. Math. Math. Phys. 1997. Vol. 37. P. 1285-1296.

2. Mastroeni G. On auxiliary principle for equilibrium problems. In: Daniele, P. et al. (eds.) Equilibrium Problems and Variational Models. Kluwer Academic Publishers, Dordrecht, 2003. P. 289-298.

3. Combettes P. L., Hirstoaga S. A. Equilibrium Programming in Hilbert Spaces. J. Nonlinear Convex Anal. 2005. Vol. 6. P. 117--136.

4. Korpelevich G. M. The extragradient method for finding saddle points and other problems. Ekonomika i Matematicheskie Metody. 1976. Vol. 12. P. 747-756.

5. Lyashko S. I., Semenov V. V., Voitova T. A. Low-cost modification of Korpelevich's methods for monotone equilibrium problems. Cybernetics and Systems Analysis. 2011. Vol. 47. P. 631-639.

6. Quoc T. D., Muu L. D., Hien N. V. Extragradient algorithms extended to equilibrium problems. Optimization. 2008. Vol. 57. P. 749-776.

7. Popov L. D. A modification of the Arrow-Hurwicz method for search of saddle points. Mathematical notes of the Academy of Sciences of the USSR. 1980. Vol. 28. Issue 5. P. 845-848.

8. Vedel Y. I., Semenov V. V. A new two-phase proximal method of solving the problem of equilibrium programming. Journal of Computational and Applied Mathematics. 2015. No. 1 (118). P. 15-23. (in Russian)

9. Lyashko S. I., Semenov V. V. A New Two-Step Proximal Algorithm of Solving the Problem of Equilibrium Programming. In: B. Goldengorin (ed.) Optimization and Its Applications in Control and Data Sciences. Springer Optimization and Its Applications, vol. 115. Springer, Cham, 2016. P. 315-325.

10. Chabak L., Semenov V., Vedel Y. A New Non-Euclidean Proximal Method for Equilibrium Problems. In: Chertov O., Mylovanov T., Kondratenko Y., Kacprzyk J., Kreinovich V., Stefanuk V. (eds.) Recent Developments in Data Science and Intelligent Analysis of Information. ICDSIAI 2018. Advances in Intelligent Systems and Computing, vol. 836. Springer, Cham, 2019. P. 50-58.

11. Vedel Y. I., Semenov V. V., Chabak L. M. About the two-stage proximal method for solving of equilibrium problems. Journal of Numerical and Applied Mathematics. 2019. No. 2 (131). P. 23-31. (in Russian)

12. Konnov I. V. Combined relaxation methods for variational inequalities. Berlin, Heidelberg, New York: Springer-Verlag, 2001. 181 p.

13. Zykina A. V., Melenchuk N. V. Finite number of iterations in the two-step extragradient method. Russian Mathematics. 2014. Volume 58. Issue 9. P. 62-65.

14. Ferris M. C. Finite convergence of the proximal point algorithm. Math. Programming. 1991. Vol. 50. P. 359-366.

15. Burke J. V., Ferris M. C. Weak sharp minima in mathematical programming. SIAM J. Control Optim. 1993. Vol. 31. P. 1340-1359.

16. Antipin A. S. On the finite convergence of processes to a sharp minimum and a smooth minimum with a sharp derivative. Differ. Equ. 1994. Vol. 30. No. 11. P.1703-1713.

17. Marcotte P., Zhu D. L. Weak sharp solutions of variational inequalities. SIAM J. Optim. 1999. Vol. 9. P. 179-189.

18. Xiu N. H., Zhang J. Z. Local convergence analysis of projection-type algorithms: a unified approach. J. Optim. Theory Appl. 2002. Vol. 115. P. 211-230. 
19. Wu Z. L., Wu S. Y. Weak sharp solutions of variational inequalities in Hilbert spaces. SIAM J. Optim. 2004. Vol. 14. P. 1011-1027.

20. Xiu N., Zhang J. On finite convergence of proximal point algorithms for variational inequalities. J. Math. Anal. Appl. 2005. Vol. 312. P. 148-158.

21. Matsushita S., Xu L. Finite Convergence of the Proximal Point Algorithm for Variational Inequality Problems. Set-Valued Variational Analysis. 2013. Vol. 21. P. 297-309.

22. Zhou J., Wang C. A note on finite termination of iterative algorithms in mathematical programming. Oper. Res. Lett. 2008. Vol. 36. P. 715-717.

23. Chabak L. M., Vedel Ya. I., Dudar V. V., Semenov V. V. Finite convergence of two-stage algorithms for solving variational inequalities. Journal of Numerical and Applied Mathematics. 2017. No. 2 (125). P. 91-99. (in Russian)

24. Konnov I. V. Application of the proximal point method to nonmonotone equilibrium problems. J. Optim. Theory Appl. 2003. Vol. 119. P. 317-333.

25. Polyak B. T. Introduction in Optimization. Moscow: Nauka, 1983. 384 p. (in Russian)

26. Bauschke H. H., Combettes P. L. Convex Analysis and Monotone Operator Theory in Hilbert Spaces. Berlin, Heidelberg, New York: Springer, 2011. 408 p.

27. Malitsky Yu. V., Semenov V. V. An extragradient algorithm for monotone variational inequalities. Cybernetics and Systems Analysis. 2014. Vol. 50. P. 271-277.

28. Semenov V. V. A Version of the Mirror descent Method to Solve Variational Inequalities. Cybernetics and Systems Analysis. 2017. Vol. 53. P. 234-243.

29. Nomirovskii D. A., Rublyov V. V., Semenov V. V. Convergence of Two-Stage Method with Bregman Divergence for Solving Variational Inequalities. Cybernetics and Systems Analysis. 2019. Vol. 55. P. 359-368.

30. Gidel G., Berard H., Vincent P., Lacoste-Julien S. A Variational Inequality Perspective on Generative Adversarial Networks. arXiv:1802.10551. 2018.

Поступила: 29.10.2019 / Принята: 08.11.2019

\section{СКІНЧЕННА КІЛЬКІСТЬ ІТЕРАЦІЙ В ДВОЕТАПНИХ АЛГОРИТМАХ ДЛЯ РОЗВ'ЯЗАННЯ ЗАДАЧ ПРО РIBНОВАГУ}

\section{Я. І. ВеДЕЛЬ, К. М. ГОЛУБєвА, В. В. Семенов}

Факультет комп'ютерних наук і кібернетики, Київський національний університет імені Тараса Шевченка, Київ, Україна, E-mail: уana.vedel@gmail.com, Katrin_G@bigmir.net, volodya.semenov@gmail.com

АнотАція. Розглянуто два двоетапні проксимальні алгоритми наближеного розв'язання задачі про рівновагу в гільбертовому просторі. В роботі доведено збіжність алгоритмів до розв'язку за скінченну кількість ітерацій при виконанні умови гостроти. Ключові словА: задача про рівновагу, біфункція, псевдомонотонність, умова гостроти, двоетапний проксимальний алгоритм, гільбертовий простір, скінченна збіжність. 